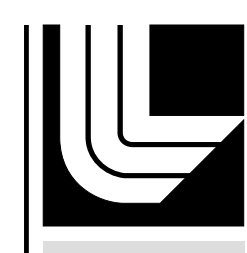

LA W RENCE LIVERM ORE NATION AL LABORATORY

Determination of effective axion masses in the helium-3 buffer of CAST

J. Ruz

November 18, 2011

IEEE

Valencia, Spain

October 23, 2011 through October 29, 2011 
This document was prepared as an account of work sponsored by an agency of the United States government. Neither the United States government nor Lawrence Livermore National Security, LLC, nor any of their employees makes any warranty, expressed or implied, or assumes any legal liability or responsibility for the accuracy, completeness, or usefulness of any information, apparatus, product, or process disclosed, or represents that its use would not infringe privately owned rights. Reference herein to any specific commercial product, process, or service by trade name, trademark, manufacturer, or otherwise does not necessarily constitute or imply its endorsement, recommendation, or favoring by the United States government or Lawrence Livermore National Security, LLC. The views and opinions of authors expressed herein do not necessarily state or reflect those of the United States government or Lawrence Livermore National Security, LLC, and shall not be used for advertising or product endorsement purposes. 


\title{
Determination of effective axion masses in the helium-3 buffer of CAST
}

\author{
J. Ruz, on behalf of the CAST Collaboration
}

\begin{abstract}
The CERN Axion Solar Telescope (CAST) is a ground based experiment located in Geneva (Switzerland) searching for axions coming from the Sun. Axions, hypothetical particles that not only could solve the strong $\mathrm{CP}$ problem but also be one of the favoured candidates for dark matter, can be produced in the core of the Sun via the Primakoff effect. They can be reconverted into $X$-ray photons on Earth in the presence of strong electromagnetic fields. In order to look for axions, CAST points a decommissioned LHC prototype dipole magnet with different $\mathrm{X}$-ray detectors installed in both ends of the magnet towards the Sun. The analysis of the data acquired during the first phase of the experiment yielded the most restrictive experimental upper limit on the axion-to-photon coupling constant for axion masses up to about $0.02 \mathrm{eV} / \mathrm{c}^{2}$. During the second phase, CAST extends its mass sensitivity by tuning the electron density present in the magnetic field region. Injecting precise amounts of helium gas has enabled CAST to look for axion masses up to $1.2 \mathrm{eV} / \mathrm{c}^{2}$. This paper studies the determination of the effective axion masses scanned at CAST during its second phase. The use of a helium gas buffer at temperatures of $1.8 \mathrm{~K}$ has required a detailed knowledge of the gas density distribution. Complete sets of computational fluid dynamic simulations validated with experimental data have been crucial to obtain accurate results.
\end{abstract}

\section{THEORETICAL MOTIVATION AND EXPERIMENTAL AXION SEARCHES}

Quantum Chromodynamics (QCD) is expected to violate CP-Symmetry, but up to now no experiment has been able to observe this effect. A possible solution to the strong CP-problem was suggested by R.Peccei and H.Quinn [1] in 1977.They explained the apparent conservation of CP in strong interactions by introducing an additional symmetry. When this new global symmetry is spontaneusly broken at a yet unknown breaking scale $f_{a}$, it gives rise to a Goldstone boson as was pointed out independently by S.Weinberg and F.Wilczek [2], [3] in 1978. This neutral pseudo-scalar is commonly referred to as the axion.

If axions exist, they could have been created in the very early universe. Nowadays however, they could still be produced in cores of stars like our Sun. Several constraints from astrophysics and cosmology have been applied, narrowing the mass range, in which the axions are still likely to exist, to a window reaching from $\mu \mathrm{eV}$ up to some meV. Experiments have attempted to detect axions in and close to the remaining mass regions. Most of them make use of the Primakoff [4] effect, which allows for a conversion of axions into photons in the presence of strong electromagnetic fields. Helioscopes [5], [6] are making use of this effect by looking for axions produced in the solar core (see Fig. 1).

J. Ruz is with the Physical and Life Sciences Department, Lawrence Livermore National Laboratory, CA, USA, emal: ruzarmendari1@1lnl.gov

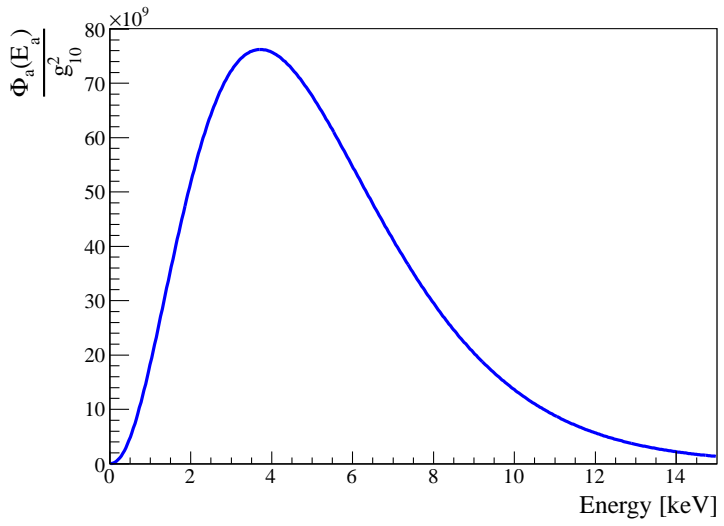

Fig. 1. Differential axion flux at the Earth's surface due to axion Primakoff production in the solar core.

\section{THE CAST EXPERIMENT}

The most sensitive existing helioscope is the CERN Axion Solar Telescope (CAST), which employs a prototype superconducting LHC dipole magnet providing a magnetic field of up to $9 \mathrm{~T}$ (see Fig. 2). CAST is able to follow the Sun twice a day during sunset and sunrise for a total of about $3 \mathrm{~h}$. At both ends of the $10 \mathrm{~m}$ long magnet, $\mathrm{X}$-ray detectors ([8], [9], [10]) have been mounted to search for photons from Primakoff conversion.

Installed on one end of the magnet, two novel MICROMEsh GAseous Structure (MICROMEGAS) detectors search for the signature of axions during sunset. On the other side of the dipole, an additional Micromegas detector and the X-ray telescope with a Charge-Coupled Device (CCD) as a focal plane detector are mounted.In light grey the cryogenics feed box (left) and return box (right) capable of keeping the magnet at $1.8 \mathrm{~K}$ to assure the superconductivity condition to achieve the $9 \mathrm{~T}$ magnetic field intensity. In each one of the extremes of the experiment there exist two ports (blue) where the different X-ray detectors are mounted.

CAST began operation in 2003 and achieved an upper limit of $g_{a \gamma}<0.88 \times 10^{-10} \mathrm{GeV}^{-1}$ at $95 \% \mathrm{CL}$ for $m_{a} \lesssim 0.02 \mathrm{eV} / \mathrm{c}^{2}$ ([12], [13]) after two years of data taking with vacuum inside the magnet bores. To extend the experimental sensitivity to larger masses CAST fills the conversion region during its second phase with a suitable buffer gas, providing the photons with an effective mass $m_{\gamma}$ [6]. The axion-photon momentum difference becomes $q=\left|m_{a}^{2}-m_{\gamma}^{2}\right| / 2 E$ and the axion mass band for which the 


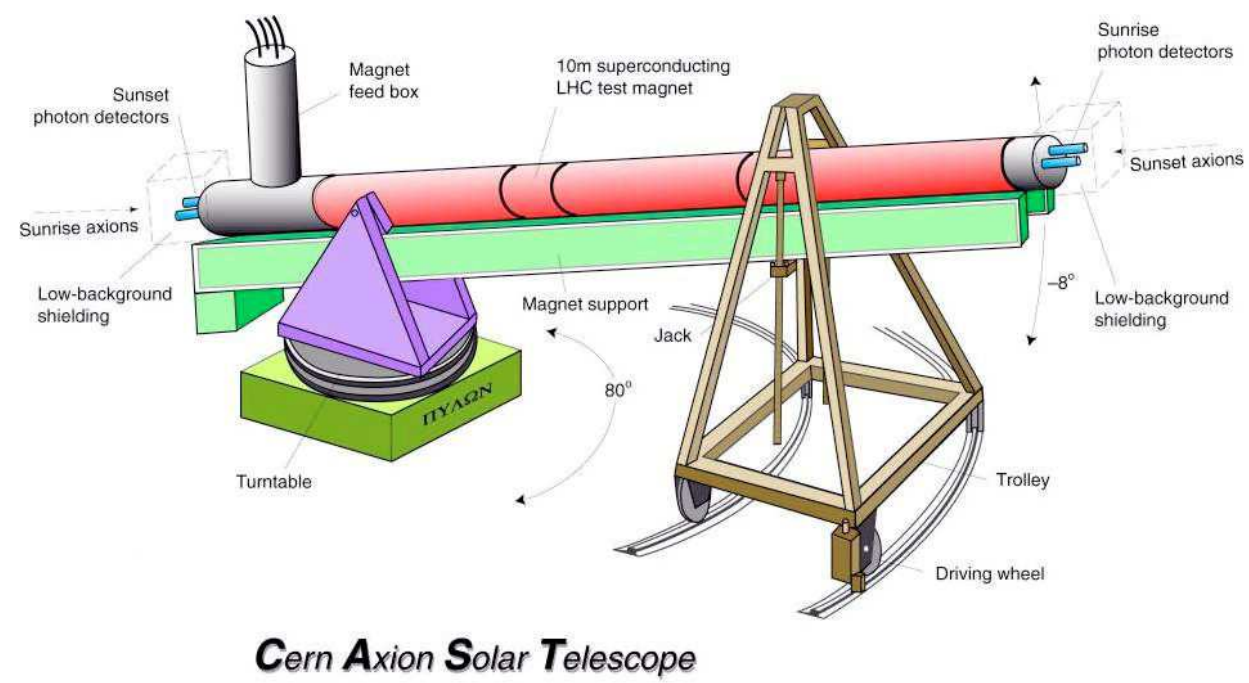

Fig. 2. Schematic view of the CAST experiment.In red the LHC dipole magnet mounted on top of the green girder attached to a turn table (purple) so that the experiment can be following the Sun in the horizontal plane. The magnet is able to move in azimuth by means of a gear system in the yellow charriot.

Primakoff based helioscope is sensitive can be extracted from the coherence condition

$$
\left(\frac{m_{a}^{2}}{e V^{2}}\right)<<\left(\frac{m_{\gamma}^{2}}{e V^{2}}\right)+2 \cdot\left(\frac{E_{a} / e V}{L \cdot e V}\right)
$$

so that, for $m_{a}$ values in the neighborhood of the chosen $m_{\gamma}$, the maximum sensitivity is restored (see Fig. 3).

During 2005 and 2006 CAST filled its magnetic field

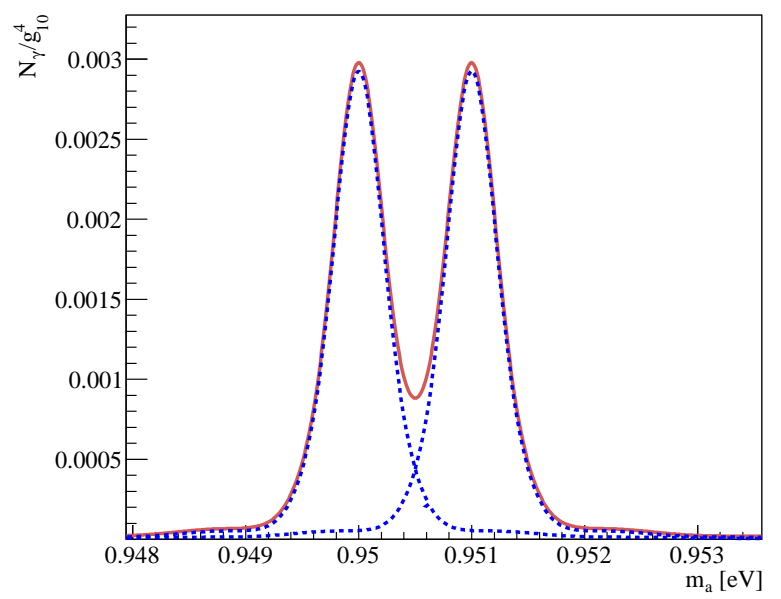

Fig. 3. Example of the coherence condition recovery at two specific axion masses. Each one of the blue dashed gaussians represent the expected photons at CAST (relative to the strength of the axion-to-photon coupling) for a single mass seting. The red line is the overall flux of photons expected from the contribution of both settings

region with helium-4 and set an upper limit on the axionto-photon coupling of $g_{a \gamma}<2.2 \times 10^{-10} \mathrm{GeV}^{-1}$ at $95 \%$ CL for $m_{a} \lesssim 0.39 \mathrm{eV} / \mathrm{c}^{2}$ [14]. After completing the data taking with helium-4 as buffer gas, the CAST experiment was upgraded in order to prepare for data taking with helium-3.
The most important upgrade was the design and installation of a sophisticated helium-3 system.

\section{A. The helium-3 system of CAST}

As shown above, capability to precise injection of gas is crucial to the successful operation of the experiment. Also, due to the high value of helium-3 gas, the system was designed as a hermetically closed gas circuit assuring full recovery of helium (see Fig. 4). The setup can be divided in five functional sections with specific purposes:

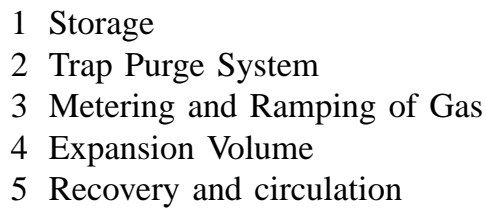

All the necessary helium for CAST physics runs is transferred to the storage volume, dimensioned to keep its gas pressure below atmospheric. Before entering the metering volumes the gas passes through two charcoal traps to remove any impurity. To scan over a wide range of axion masses CAST needs to control the helium gas density in the cold bore precisely.

This is achieved by filling the cold bore with exactly metered amounts of gas in incremental steps. The precision of the metering process is obtained by the accurate temperature control of the metering volumes, and by use of a metrology-graded pressure-measuring instrument to determine the amount of gas introduced into the cold bore. The measurement of the amount of gas transferred to the cold bore is calculated by accurately measuring the pressure decrease on the metering volumes that are maintained at a constant temperature of $309.15 \mathrm{~K}$ by means of a thermostatic bath with $\pm 0.01 \mathrm{~K}$ temperature stability.

A single full-scale (FS) pressure measurement in the metering volume can be made with an accuracy of $42 \mathrm{ppm}$, taking into account the precision of the MKS Baratron gauge, and the drift of its zero and span under ambient temperature variation. 


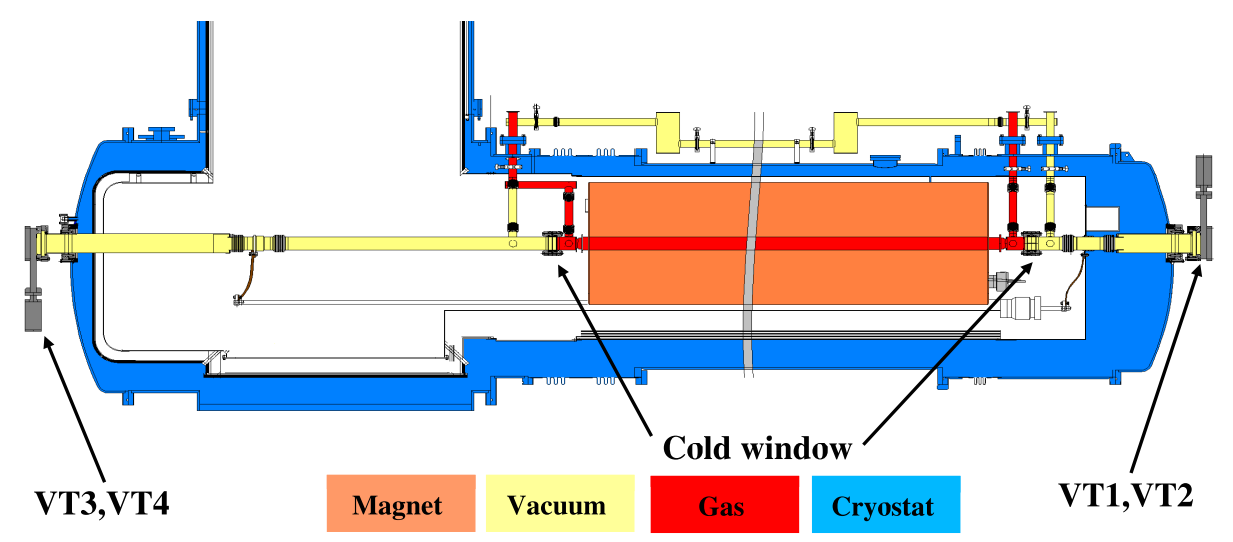

Fig. 4. View of the vacuum and Helium system scheme of the CAST experiment. The cryostat of CAST offers four ports (VT1-VT4) for the installation of the X-ray detectors. The X-ray windows are placed such that are able to keep the Helium gas (red) within the magnet region of the cryostat (orange). Note that between the windows and the ports $t$ which the detectors are connected there is vacuum (yellow).

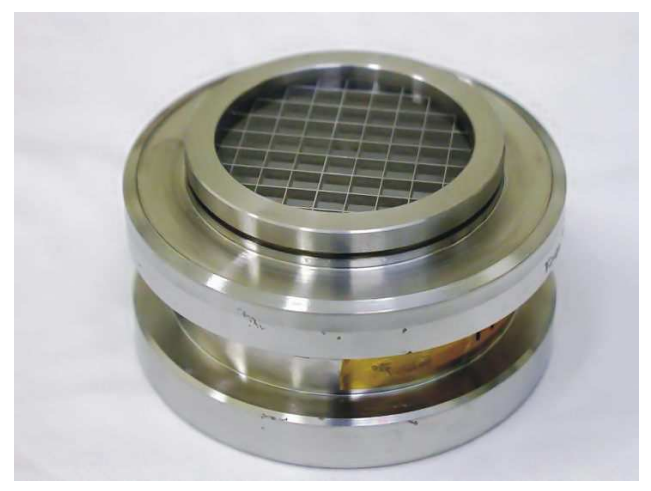

Fig. 5. View of one of the four X-ray windows before its installation inside the cold bore of CAST.

The transfer of gas to the cold bore is performed using a cryogenic valve. The temperature of the gas along the cold bore tubes should be kept stable during run conditions. The gas is confined to the cold bore region of the magnet by thin X-ray windows composed of a thin layer of polypropylene glued on top of a stainless steel grid (see Fig. 5). Heaters on the window flanges were installed to make periodical bakeout of gases adsorbed on the window foils. The use of $15 \mu \mathrm{m}$ polypropylene allows for a $95 \%$ transmisson at $4.2 \mathrm{keV}$.

To avoid thermoacoustic oscillations, the warm pipes linking to room temperature volumes are isolated during normal operation by means of cryogenic valves, i.e. a check valve and a shut-off valve, which are closed during normal operation, and open only during a quench ${ }^{1}$ when evacuating the cold bore.

In case of a quench, the temperature of the cryostat increases rapidly. If the cold volume remains closed, the gas pressure will rise proportionally. This puts the integrity of the X-ray windows in danger, and requires that the gas is sufficiently fast evacuated to keep the pressure below the safe operating pressure of the windows ( $1.5 \mathrm{bar})$. A cryogenic check valve opens when the differential pressure reaches $30 \mathrm{mbar}$, whereas the cryogenic valve will open when triggered by the quench protection system. During the quench, the gas is safely evacuated from the cold bore to an expansion volume via two electro-pneumatic valves. The expansion volume initially under vacuum thus acts as a buffer volume for the gas coming out of the cold bore during a quench. Two electro-pneumatic valves, opened by quench-trigger signal activation, and the gas is led to an expansion volume. The pressure reached in case of quench should not exceed 1.2 bar to have safety margin.

The helium system of CAST is integrated in two independent computers. The PLC (Programmable Logic Controller) computer runs the PVSS Unicos software which commands all the valves and pumps of the helium system, while the Labview computer calculates the density settings and monitors all temperatures and pressure variations. Any valve manipulation is done via the PLC computer. Several custom made routines in the PLC allow to set the desired gas density in the cold bore of CAST or, if necessary, the recovery of the gas in case of a quench. Those routines include the appropiate programing of valve and pump sequences as well as safety interlocks.

\footnotetext{
${ }^{1}$ A quench is an interesting phenomenom related to the sudden change from "super" to "normal" conductive state of certain magnet coils materials. The presence of minimal impurities in super conductive materials might generate instant electric resistivities. That provokes a violent warming of localized coil regions due to the Joule effect. CAST's coils use $13 \mathrm{kA}$ current to achieve $9 \mathrm{~T}$ magnet field intensity and uses super fluid helium-4 to refrigerate the magnet coils of the dipole. When a quench happens at CAST, the heat generated is dissipated by the liquid helium that instantly gasifies. In order to preserve the integrity of the system, the gas is released from the cryostat warming up the magnet bores.
} 


\section{EFFECTIVE PHOTON MASSES AT CAST}

The electron density $\left(n_{e}\right)$ of the gas filling the cold bore drives the physics of the axion-photon conversion at CAST. The effective mass of the backconverted photon can be obtained as:

$$
m_{\gamma}^{2}=\omega_{p}^{2}=4 \pi n_{e} \frac{e^{2}}{m_{e}}=4 \pi n_{e} r_{e}
$$

where $\omega_{p}$ is the plasma frequency and $r_{e}$ the electron radius. Helium-3 and helium-4 are equivalent regarding the effective photon mass, since they have the same number of electrons per molecule even though they have different nuclear masses and therefore densities.

CAST data taking procedure requires step ramping over different densities of gas filling the cold bore in order to allow the search of different axion masses.

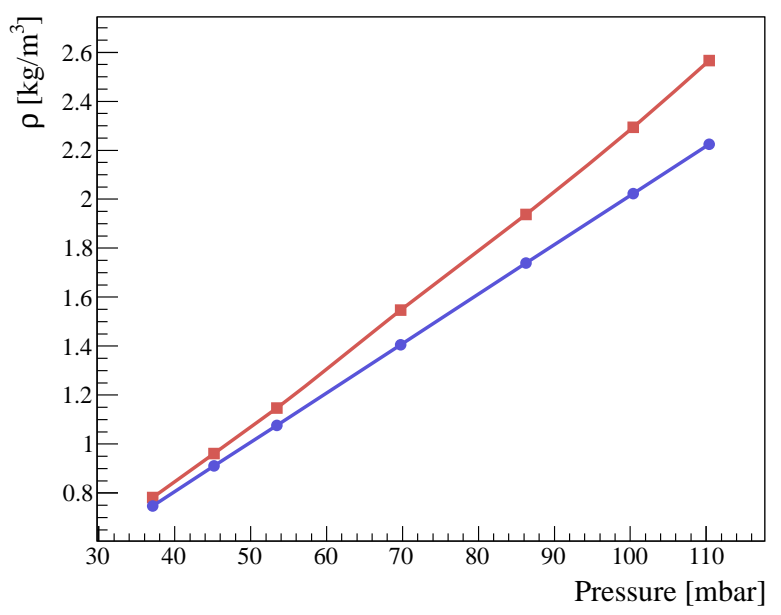

Fig. 6. Comparisson between the densities achieved in the center of the coldbore at CAST for a given pressure for a real gas (red) and the ideal gas (blue) equation of states.

Achieving the desired density is a demanding task that requires computational fluid dynamic simulations of the actual system including different physical phenomena such as the hydrostatic effect, convection and buoyancy.

A close look to the results shows that the density achieved in the center of the cold bore is proportional to the pressure of helium-3 in the system (see Fig. 6). Under extreme conditions, such as high pressures or extremely low temperatures, some gases show deviations from the ideal gas law. In particular, at extremely low temperatures, gas molecules are moving relatively slower than at higher temperatures. So slow, in fact, that they no longer travel past each other rapidly enough to not experience the attraction or repulsion that might exist. At low temperatures, intermolecular forces between gas molecules are no longer negligible.

There are several equations of state capable of describing such phenomena. Among them, the Peng-Robison [7] equation of state describes not only the fact that the molecules of gas can compress or interact with each other, but also their nonspherical nature. Its use can be extended in thermodynamic applications and it is best suited to accurately describe the actual conditions at CAST (see Fig. 7).

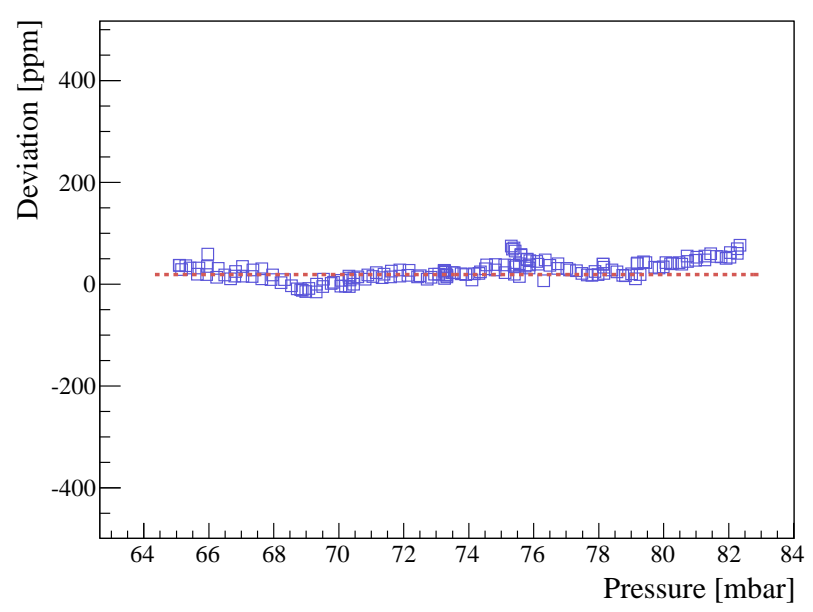

Fig. 7. Deviation in ppm between the mass present in the CAST system at different pressures relative to the amount of mols according to CFD simulations.

The probability for Primakoff conversion of axions depends on the density of electrons present in the system as well as paramenters such as the intensity of the magnetic field, and the length of the path that axions travel thtough the media:

$P_{a \rightarrow \gamma}=\left(\frac{B_{\perp} g_{a \gamma}}{2}\right)^{2} \frac{1}{q^{2}+\Gamma^{2} / 4}\left(1+e^{-\Gamma L}-2 e^{-\Gamma L / 2} \cos q L\right)$

where $B_{\perp}$ is the intensity of the magnetic field perpendicular to direction of the incident axion, $g_{a \gamma}$ the axion-to-photon coupling strength,$q$ is the axion-photon momentum tranfer, $L$ the length of the magnetic field and $\Gamma$ the gas absorption coefficient [6].

As can be seen from the performed simulations, the region with constant density depends on the convection currents of gas between the X-ray windows and the inner regions of the cold bore (see Fig. 8). This effect translates to an effective magnetic length in which the axions may convert back into photons. Assuming a density homogeneity better than a $\mu \mathrm{gr} / \mathrm{cm}^{3}$ in the inner region of the cold bore we can express the effective magnetic length as function of the pressure of the 3-helium gas present in the magnet bores of CAST. It is clear that the ratios will strongly depend on the experimental setup.

Although the superfluid helium that cools down the CAST magnet assures stable temperatures in the inner regions of the cold bore, there are several factors causing variations. Both the temperature of the X-ray windows and the density of the gas present in the system influence the convection currents. These effects change the effective length of the magnetic 


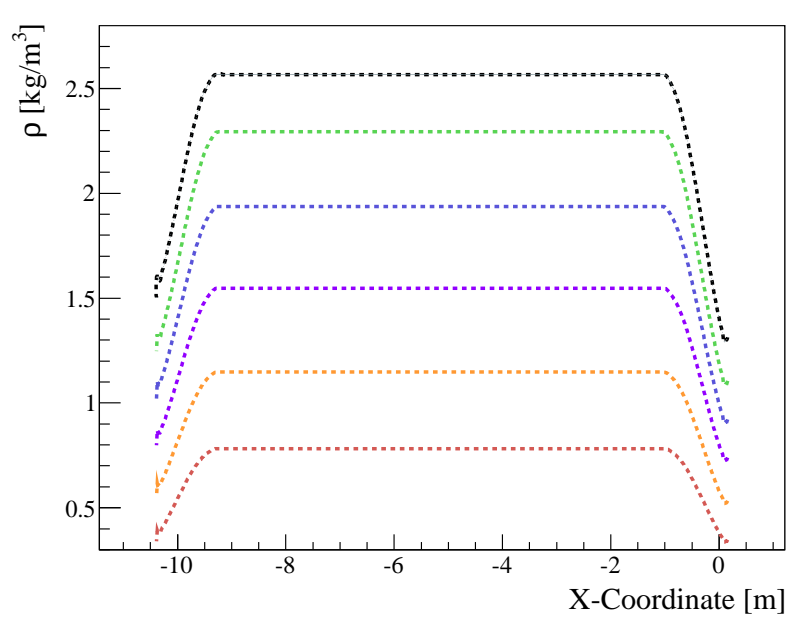

Fig. 8. Simulated density profile along the cold bore of CAST for different pressures in the system. The X-ray windows are placed at both extremes of the cold bore distant $\sim 10 \mathrm{~m}$ from each other. In the figure, the magnetic region limits at coordinates $-0.496 \mathrm{~m}$ and $-9.731 \mathrm{~m}$. Profiles obtained using the Peng-Robinson state equation for different pressures of helium-3 in the cold bore. In the figure you can observe in different colors the profiles obtained for 35.85 mbar (red dashed lowermost line), and rising in density, 51.64 mbar (orange dashed line), 67.45 mbar (purple dashed line), 83.34 mbar (blue dashed line), 97.02 mbar (green dashed line) and 106.7 mbar (black dashed uppermost line) .

field. Heating the X-ray windows, for instance, while a fixed amount of gas is present in the cold bore will increase the final pressure of the system. It will also increase the convection currents between the X-ray windows and the helium gas. An increase of the temperature of the X-ray windows will translate as a shortening of the effective magnet length.Keeping the $\mathrm{X}$-ray windows at $15 \mathrm{~K}$ (i.e. no extra heating applied to the X-ray windows), shows a linear dependence of the effective length of the magnet with the pressure achieved in the system:

$$
L_{e f f}[m]=8.611-0.021 \cdot P_{@ 1.8 K}
$$

Tilting a $10 \mathrm{~m}$ magnet full of helium-3 while tracking the Sun has also some consequences to the effecive photon mass tuned in the cold bore of the experiment. Gravitational forces are capable of generating a gradient of densities in the gas column of the magnet region.

Such an effect can be corrected by applying an effective density to the whole gas column, since the movement of the system is symmetric with respect to the polar angle. CAST monitors the pressure of the system continuously. A correction of the kind

$$
\rho(\theta)=[1+\alpha(\theta)] \times \rho_{C F D_{0}}
$$

can be applied, where $\alpha(\theta)$ is a factor that accounts for the equivalent density in the center of the cold bore relative to the density at $\theta=0 \mathrm{deg}$. This translates to an effective photon mass that now depends not only on the pressure of the

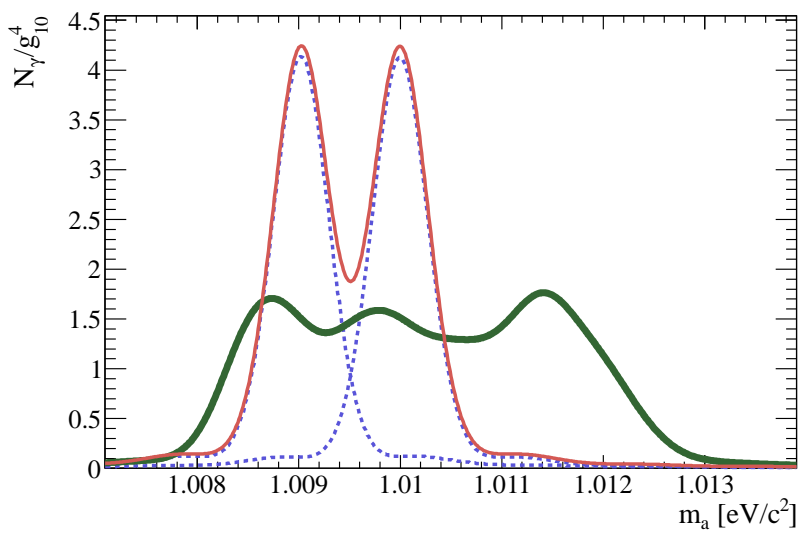

Fig. 9. Expected flux of photons at CAST for different axion masses scanned during a tracking. In dashed blue, gaussians for an ideal setup in which the density in the cold bore is kept constant for all the tracking. The contribution of both gaussians to the final flux adds to the solid red line. In thick green, the effective axion masses scanned during a real tracking at CAST. A smear over a wider axion mass region is the result from the hydrostatic effect of the magnet tilting while tracking.

system, but also on the polar angle at which the experiment is placed:

$$
m_{\gamma}(\theta, P)=\beta \sqrt{\rho(\theta)}=\beta \sqrt{[1+\alpha(\theta)] \times \rho_{C F D_{0}}}
$$

where $\beta$ is a constant for the helium-3 gas.

The number of expected photons arising from the Primakoff conversion inside the magnet of CAST, can be accurately calculated by taking into account the fact that the density profile is homogeneous for a given effective length. Using the total exposure to the Sun together with all the other experimental parameters, such as an instant pressure in the coldbore at different inclinations, the amount of gas present in the system at any moment and boundary conditions such as temperatures along the cold bore and confining X-ray windows, expectations can be calculated (see Fig.10).

\section{LATEST RESUltS OF CAST}

Since 2008, CAST has been searching for axions with relatively higher masses. Up to now, the experiment has already looked for axions with masses up to $1.18 \mathrm{eV} / \mathrm{c}^{2}$ (see Fig. 10) completing its helium-3 physics program. In the exclusion plot (see Fig. 11) we present our most recent results for axion masses up to $0.65 \mathrm{eV} / \mathrm{c}^{2}$ stablishing an upper limit of $g_{a \gamma} \lesssim 2.3 \times 10^{-10} \mathrm{GeV}^{-1}$ at $95 \%$ C.L [15]. The data analysis for higher masses is ongoing and the goal of $1.18 \mathrm{eV} / \mathrm{c}^{2}$ will be reached in future publications.

\section{CONCLUSIONS AND OUTLOOK}

The final results for the vacuum and helium-4 data are displayed in Fig.11 together with the first results for the 


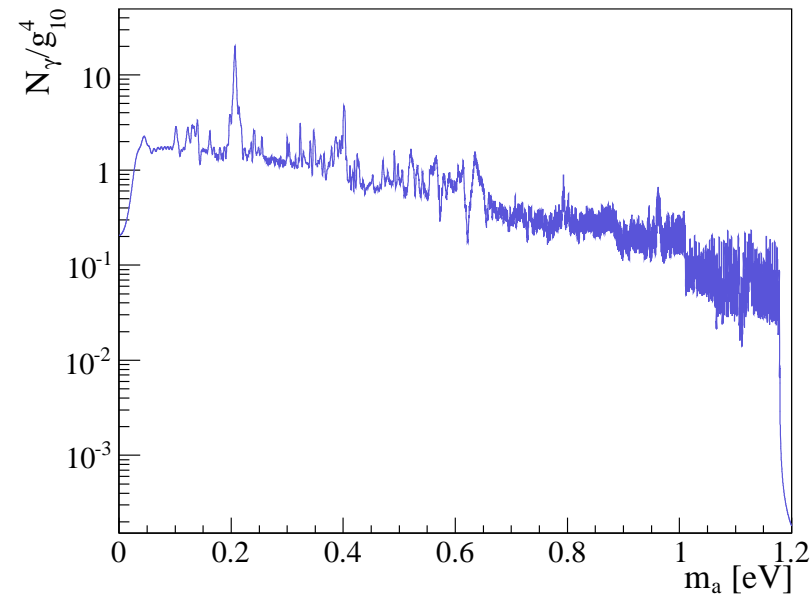

Fig. 10. Exposure of CAST to the different axion masses.

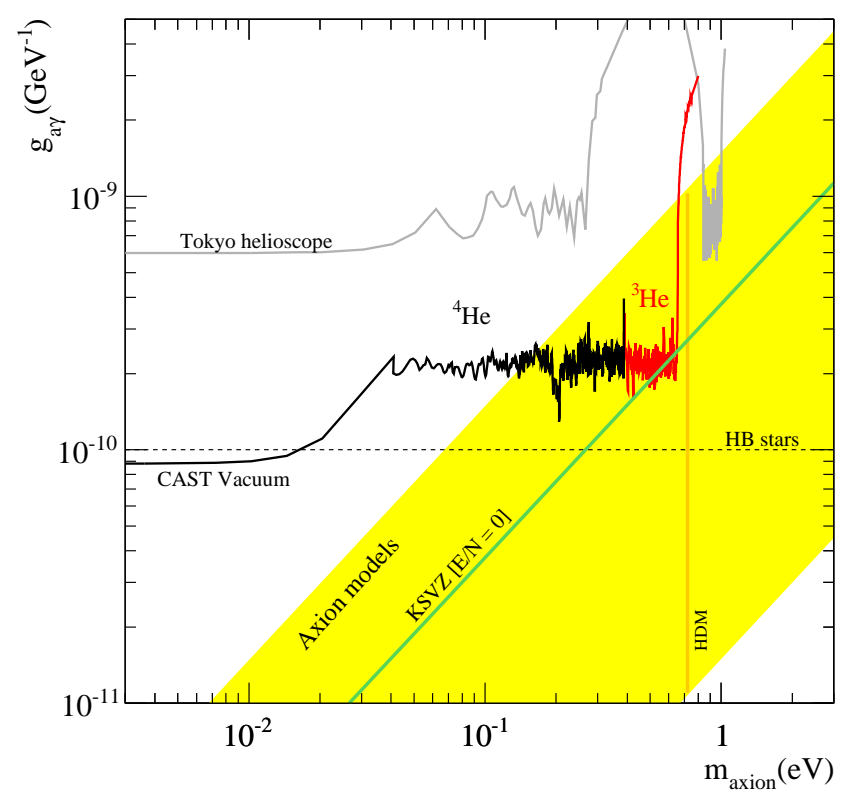

Fig. 11. CAST exclusion plot of the axion-to-photon coupling constant at 95\% CL for all data collected in Phase I and Phase II [15]. The achieved limit of CAST is compared witht he Horizontal Branch (HB) star limit [11]. The yellow band represents the theoretical QCD axion models and the green solid line corresponds to the KSVZ model with $\mathrm{E} / \mathrm{N}=0$. In black the results obtained during the first phase of CAST [12],[13] together with the results from the helium-4 data taking period [14]. In red the novel limits coming from the analysis of one year run with helium-3 during 2008[15].

helium-3 run, during which axion masses up to $1.2 \mathrm{eV} / \mathrm{c}^{2}$ were being studied. Currently, CAST is extending its axion search even further into the unexplored regions of the favored axion models. The analysis of these data is ongoing and latest results are presented in this contribution. These results include also the status of the current gas simulations used to determine the gas density along the magnetic field region which is a critical factor in obtaining accurate results.

\section{ACKNOWLEDGEMENT}

This work was performed, in part, under the auspices of the U.S. Department of Energy by Lawrence Livermore National Laboratory under Contract DE-AC52-07NA27344. The support of the Laboratory Directed Research and Development Program is gratefully acknowledged.

\section{REFERENCES}

[1] R. D. Peccei and H. R. Quinn, Physical Review Letters 38 (1977) 1440.

[2] S. Weinberg, Physical Review Letters 40 (1978) 223.

[3] F. Wilczek, Physical Review Letters 40 (1978) 279.

[4] H. Primakoff, Physical Review Letters 81 (1951) 899.

[5] P. Sikivie, Physical Review Letters 51 (1983) 1415 [Erratum ibid. 52 (1984) 695].

[6] K. van Bibber et al, Physical Review D. 39 (1989) 2089.

[7] Peng, D.Y., and Robinson, D.B, Industrial and Engineering Chemistry: Fundamentals 15: 59-64 (1976)

[8] D. Autiero et al., New Journal of Physics 9 (2007) 171.

[9] P. Abbon et al., New Journal of Physics 9 (2007) 170.

[10] M. Kuster et al., New Journal of Physics 9 (2007) 169.

[11] G. G. Raffelt, Stars as Laboratories for Fundamental Physics, University of Chicago Press, (1996).

[12] K. Zioutas et al. [CAST Collaboration], Physical Review Letters 94 (2005) 121301.

[13] K. Zioutas et al. [CAST Collaboration], Journal of Cosmology and Astroparticle Physics 04 (2007) 010.

[14] K. Zioutas et al. [CAST Collaboration], Journal of Cosmology and Astroparticle Physics 02 (2009) 008.

[15] K. Zioutas et al. [CAST Collaboration], arXiv:1106.3919v1 [hep-ex] 20 Jun 2011. Accepted to be published in Physical Review Letters. 